\title{
The Effect of Online Solution-Focused Support Program on Parents with High Level of Anxiety in the COVID-19 Pandemic: A Randomized Controlled Study
}

\author{
Mürşide Zengin ${ }^{1}$, Ceyda Başoğul ${ }^{1}$, and Emriye Hilal Yayan ${ }^{1}$ \\ ${ }^{1}$ Affiliation not available
}

November 1, 2021

\begin{abstract}
Aim:The aim of this study was to determine the anxiety levels of parents with children aged 3-6 years due to the Coronavirus Disease-2019 (COVID-19) pandemic and to examine the effects of Solution-Focused Support Program (SFSP) applied to parents with high level of anxiety.

Methods: The study was conducted as a parallel-group, randomized controlled design. The sample of the study consisted of 77 parents who were randomly assigned to the experimental and control groups (control group $\mathrm{n}=40$; intervention group $\mathrm{n}$ $=37$ ). One session of online SFSP was applied to the intervention group each week and 4 sessions were applied in total. No intervention was applied to the control group. The data were collected using introductory information form and State-Trait Anxiety Inventory (STAI). Statistical Package for the Social Sciences (SPSS) programme and Analysis of Moment Structures (AMOS) 23 application were used in the analysis of the data. Structural Equation Modeling (SEM) was used to examine a hypothesized model that SFSP has both direct and indirect effects on anxiety levels of parents.

Results: The state and trait anxiety mean scores of the intervention group decreased compared to the pre-intervention mean scores after the implemented programme. While this difference between state anxiety scores was statistically significant (p[?] $.001)$, the difference between trait anxiety scores was not statistically significant $(\mathrm{p}>.05)$. There was no statistically significant difference between the pre-test and post-test STAI total scores of the control group.
\end{abstract}

Conclusions: In the study, it has been found that SFSP applied to parents with high level of anxiety is an effective method in reducing the state anxiety levels of parents.

Clinical trial number: NCT04609722 (Registration date: 30.10.2020)

\section{What's Known}

- It is stated that the direct and indirect psychological and social effects of the Coronavirus Disease-2019 (COVID-19) pandemic are widespread and it will adversely affect mental health.

- Parents experience many stress factors such the closure of schools and care facilities, continuation of education at home, parents' being unable to hug their children with the fear of contamination and obligation to go to work.

Solution-Focused Support Program (SFSP) is a short, resource-oriented and goal-oriented therapeutic approach that helps individuals change by creating solutions.

\section{What's New}

This is the first study which investigates the effect of SFSP applied to parents with high level of anxiety in the COVID-19 pandemic on reducing anxiety.

- It has been found that SFSP applied to parents with high level of anxiety is an effective method in reducing the state anxiety levels of parents. 
Considering the advantages of applying SFSP in a short time and its rapid effect, it is recommended that SFSP should be applied to parents in order to minimize their anxiety levels during pandemic.

\section{Introduction}

Coronavirus Disease-2019 (COVID-19) spread rapidly across the world and caused a pandemic. ${ }^{1}$ The COVID19 pandemic not only affected individuals in social, economic and mental areas, but also a significant change occurred in their lifestyle. ${ }^{1,2}$

It is stated that the direct and indirect psychological and social effects of the COVID-19 pandemic are widespread and it will adversely affect mental health now or in the future. ${ }^{2}$ A serious anxiety response occurs in people living in the regions where the pandemic is experienced. ${ }^{3}$ Studies have revealed that anxiety, depression and sleep problems are common in adults during the COVID-19 pandemic. ${ }^{4-6}$ Previous studies have examined the mental health effects associated with other infectious disease outbreaks including the 2003 severe acute respiratory syndrome (SARS) epidemic, ${ }^{7} 2009$ novel influenza A (H1N1) pandemic, ${ }^{8}$ and 2014-2016 Ebola pandemic. ${ }^{9}$ As a matter of fact, the detection of symptoms such as anxiety, depression, and post-traumatic stress disorder in those affected by previous pandemics shows that similar problems may be experienced in the current process.

The COVID-19 pandemic ushered in enormous disruptions to family life. ${ }^{10}$ In addition to a sudden change in work life, family life and social life routines, economic problems and fear of pandemic negatively has affected the well-being of parents. ${ }^{11}$ During this period, parents experience many stress factors such the closure of schools and care facilities, continuation of education at home, parents' being unable to hug their children with the fear of contamination and obligation to go to work. Parents also may feel fear and uncertainty about how to keep their families safe. ${ }^{12}$ All these issues may reveal mental problems in parents and cause existing problems to get worse at the same time. In addition, it is predicted that the COVID-19 pandemic will not only cause mental problems but also cause an increase in future anxiety. ${ }^{13}$ High levels of anxiety in parents are also important in terms of causing an increase in children's anxiety ${ }^{10}$ and increasing the risk of harsh parenting and maltreatment. ${ }^{14}$ The psychosocial effects of the pandemic may occur over a long period of time on the parent and child population. For this reason, it is critical to minimize the negative effects that may be experienced in the long term by implementing early interventions to reduce the level of anxiety.

Solution-Focused Support Program (SFSP) is a short, resource-oriented and goal-oriented therapeutic approach that helps individuals change by creating solutions. ${ }^{15}$ It is a therapy method that focuses on individuals' becoming aware of their potential revealing their strengths. ${ }^{16,17}$ Focusing on the solution can increase the self-confidence of individuals, their capacity to produce solutions, and their ability to see their strengths and positive aspects. ${ }^{18}$ It is reported in the literature that short-term therapies provide faster benefits than long-term psychotherapies. ${ }^{19}$ For this reason, it is thought that short-term therapies will be applied in a short time and have a rapid effect in reducing the anxiety of parents during the pandemic. In the light of this information, it is aimed to determine the anxiety levels of parents with children between the ages of 3-6 in the COVID-19 pandemic and to examine the effects of online SFSP, which is planned to be given to parents with high anxiety levels in the study. This study differed from previous studies as it was carried out with the participation of parents living in the South-eastern Anatolia Region of Turkey. In this study, identifying parents' anxiety level and effects of online SFSP by applying a unique measurement instrument was also an important aim.

Research hypotheses are as follows;

$\mathbf{H}_{\mathbf{1}}$ SFSP is an effective method in reducing the state anxiety levels of parents with children aged 3-6.

$\mathbf{H}_{2}$ SFSP is an effective method in reducing trait anxiety levels of parents with children aged 3-6.

\section{Methods}

Study Design 
The study was conducted using a randomized controlled research design. A parallel trial design was used describing an experimental group and a control group as the two arm. This study was guided by the Consolidated Standards of Reporting Trials (CONSORT) checklist. $^{20}$

\section{Participants}

The study was carried out in a province located in the South-eastern part of Turkey between June-December 2020. The study population consisted of parents with children aged 3-6 years. The parents of this age group formed the sample of the study, considering that children in this age group could not attend nursery school due to the pandemic, could not properly transfer their energies due to staying at home all day, and were more dependent on their parents for care, education, and the establishment and maintenance of individual games.

In line with the inclusion criteria, parents with children aged 3-6 years who attended a day care centre or nursery during the pre-pandemic period, who had had a pre-test anxiety scale score above 40, a computer or internet infrastructure at home and volunteered to participate in the study were included in the research. Parents who had communication problems, had a pre-test anxiety scale score below 40, not volunteered to participate in the study, used drugs for anxiety or depression, did not have a computer or internet environment, did not attend at least one session were excluded from the study.

\section{Sample Size}

The sample size was calculated based on the study conducted by Ylldırm and Aylaz (2019) to determine the effect of solution-focused group counselling on the anxiety levels of individuals with nutrition disorder. According to the results of this study, the post-process STAI anxiety score was reported as $40.45 \pm 9.22$ in the intervention group and $46.73 \pm 8.83$ in the control group. The influence quantity of the study was determined as $\mathrm{d}=0.839 .^{21}$ The sample size was calculated using $\mathrm{G}^{*}$ Power $^{22}$ with a large effect size of 0.84 , probability of alpha error .05, a power (1- $\beta$ ) of 0.80 to perform two independent group model. Minimum sample size was 68 , totally. Considering the possible data loss during the study process, it was decided to conduct the study with a total of 90 parents, 45 parents in both groups.

Pre-test was applied until 90 parents with high anxiety levels (whose STAI-S and STAI-T scores were above 40) and volunteering to participate in the study were reached. The snowball sampling method was used in the study due to quarantine and isolation measures. With the snowball sampling method that was used in the study, a few participants were contacted initially, other participants were contacted with the help of these few participants, and the sample was formed in a sequential fashion. Pre-test was applied to 102 parents in this process. Parents who did not meet the inclusion criteria were not included in the study $(\mathrm{n}=12)$.

Parents who were included in the study $(\mathrm{n}=90)$ were assigned to intervention $(\mathrm{n}=45)$ and control $(\mathrm{n}=45)$ groups randomly using a randomization program. Eight parents from the intervention group and five parents from the control group were not included in the evaluation process for various reasons (not completing the sessions, not completing the final test). Thus, a total of 77 parents were included in the study, 37 of whom were in the intervention group (SFSP) and 40 of whom were in the control group (no intervention) (Fig. 1).

\section{Randomisation}

The randomisation sequence is performed in permuted blocks of variable sizes stratified for trial site using centralised, concealed allocation. The randomisation sequence is generated 1:1 (intervention/control) ratio to each arm using the online randomisation software 'Sealed Envelope' (https://www.sealedenvelope.com/).

\section{Measurements}

A descriptive information form which was prepared by the researchers in line with the literature $\mathrm{e}^{3,10,21,23,24}$ and the State-Trait Anxiety Inventory were used for collecting the data of the study.

Introductory Information Form consists of 21 questions for evaluating the socio-demographic characteristics of the parents (age, gender, socioeconomic status, etc.) and their views and behaviours during the 
pandemic period (living areas affected by the pandemic, coping behaviours, etc.).

\section{State-Trait Anxiety Inventory (STAI)}

It was developed by Spielberg et al. in 1970 in order to measure the trait and state anxiety levels of individuals and adapted to Turkish by Öner and Le Compte (1983). ${ }^{25}$ State-Trait Anxiety Inventory includes a total of forty items which are the State Anxiety Scale consisting of 20 items and the Trait Anxiety Scale consisting of 20 items. The State Anxiety Sub-Scale (STAI-S) requires the individuals to describe how they feel at a certain time and under certain conditions while the Trait Anxiety Sub-Scale (STAI-T) requires the individuals to describe how they feel in general. In the State Anxiety Scale, the response choices collected in four classes are (1) Not at all, (2) somewhat, (3) Moderately so, and (4) Very much so while the response choices for Trait Anxiety Scale are (1) Almost never, (2) Sometimes, (3) Often and (4) Almost always. The scores obtained from the scales theoretically vary between 20 and 80 . High score indicates high level of anxiety while low score indicates low level of anxiety. Reliability coefficient of the scale was found between 0.94 and 0.96 for the "State Anxiety Scale" and between 0.83 and 0.87 for the "Trait Anxiety Scale". Based on these data, it is understood that Turkish scales have high item homogeneity and internal consistency. ${ }^{26,27}$ For this study, Cronbach alpha coefficients for state and trait anxiety subscales were found to be 0.90 and 0.85 , respectively.

\section{Data Collection}

The data of the study were collected through a questionnaire form which was created through the GoogleForms programme. Google Forms is an online application through which only the participants and researchers can have access to data. Thus, the confidentiality of study data was ensured. Besides, the survey form designed not to allow any respondent to submit the form before answering all questions prevented the respondents from submitting a survey form with missing data. Duplicate entries were prevented by allowing one submission for each participant's Google account. Firstly, the parents who agreed to participate in the study were asked to complete the informed consent form designed in Google Forms. In the first telephone conversation with the parents assigned to the experiment and control groups, information was given about the study. The forms were sent to the parents as an online link and they were asked to fill the form. In the information form, a space was provided for the participants to write their phone numbers voluntarily. In this way, parents with high levels of anxiety were contacted by phone and asked whether they would like to participate in the online SFSP program. Online SFSP was applied to the parents who wanted to participate in the program via video conferencing. The programme was prepared and implemented by a researcher holding a "Solution-Focused Consulting Approach" certificate. SFSP provides short-term consultancy that focuses on the solution rather than the problem encountered. The programme was implemented as four sessions each of which lasted 40-50 minutes in average. We applied the SFSP with four session because it was reported that is most effective in the 3 - to 5 -session range. ${ }^{23,28}$ Each session was conducted with the participation of six to eight parents using video conferencing method. The same participants were in the group all sessions. Interviews were held at the time determined with the participants and when they were available. Data collection forms were applied to the intervention group after the programme to the control group approximately one month after the pre-test and the data collection was completed for the study. During the study period, participants in both groups received no pharmacological or non-pharmacological treatment for decreasing anxiety or increasing mental health.

\section{Intervention}

Firstly, national and international literature review was conducted in order to develop this programme. ${ }^{23,29-35}$ Expert opinions were also asked in the development of the programme. Framework of the programme, which was developed within the scope of this study, was prepared depending on the basic philosophy, principles and techniques of the solution-oriented approach. The sessions of the prepared programme are briefly as follows:

Session 1: Getting acquainted (In this session, it was aimed to meet the group members, explain the goals and rules of the group, give brief information about the basic philosophy and principles of the solutionoriented approach, explain the programme process, share thoughts about the pandemic, create positive goals, discover what they do well and make a connection between now and the future. Homework: Explaining the 
concerns about the pandemic).

Session 2: Miracle Question - Awareness of Exceptions and Alternatives (In this session, it was aimed to raise awareness for change, create positive change expectation in group members, make group members think about solutions, make them aware of their effects on solutions and their own resources, Homework: journal keeping, positive thinking techniques)

Session 3: Coping Skills (In this session, it was aimed to discuss group members' ability to cope with obstacles in achieving the goal, discuss solutions by recognizing useful things, try more useful things and be able to transfer them. Homework: relaxation exercises, breathing exercises).

Session 4: Positive Design of the Future and Finalization (In this session, it was aimed to design the future in a positive way, discuss how to reach a positive future and evaluate the gains from the group process for finalization, receive feedback on the programme process, make an overall evaluation of the group process and finalize the group process).

\section{Data analysis}

Statistical Package for the Social Sciences (SPSS) 22 programme (v. 22.0; SPSS Inc., Chicago, IL) was used for the analysis of the research data. Percentage and mean were used to examine the distribution of sociodemographic characteristics and chi-square test was used to examine difference between the groups. The normality of the parameters in the study data was checked with the Shapiro-Wilk test. Since the data provided parametric assumptions, Paired t test was used to compare the means of scales between the intervention and control groups. To determine the effect of SFSP on state and trait anxiety levels the "Structural Equation Modeling (SEM)" was used in Analysis of Moment Structures (AMOS) 23 application. An alpha level of .05 was used for all statistical tests. Cronbach alpha was used to evaluate the internal consistency of the scales.

\section{Ethical Consideration}

Ethical permission was obtained from the Scientific Research and Publication Ethics Committee of the Inönü University with the resolution number 02.06.2020/751. Since the inclusion of the human phenomenon in the study requires the protection of individual rights, "Informed Consent" condition was fulfilled as an ethical principle. In addition to explaining "Autonomy" principles, the individuals participating in the study were informed that they could withdraw from the research before the data was started to be collected, their identity information would be kept confidential and not shared with third parties in order to comply with the "Protection of Privacy" ethical principles. After the last scale was administered to the experimental and control groups, group counseling was offered to the individuals in the control group upon their wishes.

\section{Results}

In present study, 102 parents were assessed for eligibility. 90 parents who meet the inclusion criteria were randomized as 45 individuals in each groups (Fig. 1). The socio-demographic characteristics of the parents participating in the study are presented in Table 1.

The average age of the parents is $33.51 \pm 6.53,54.5 \%$ of them are in the 30-39 age group. The majority of the parents $(77.9 \%)$ were female, university graduates $(51.9 \%)$, had middle income (58.4\%) and the majority $(90.9 \%)$ had no illness. In addition, about half of the parents $(51.9 \%)$ were working during the pandemic period. There was no statistically significant difference between the intervention and control groups in terms of sociodemographic characteristics before the intervention $(\mathrm{p}>.05)$ (Table 1).

When the issues that cause the parents, who participated in the study, to be worried were examined; it was seen that $64.9 \%$ of the parents stated that they were afraid that their child could be sick and that their child's being away from school negatively affected his/her mental health. In addition, $58.4 \%$ of the parents stated that their child became aggressive because he/she was always at home.

It was determined that the majority of the parents, who participated in the study, had access to information 
about the pandemic via TV (34\%) and social media (29\%). It was determined that the social life of $93.5 \%$ of the parents, the family life of $66.2 \%$ of the parents, the work life of $55.8 \%$ of the parents, the economic status of $54.5 \%$ of the parents and the sexual life of $15.6 \%$ of the parents were affected. When the parents' coping behaviours during the pandemic period were examined, it was determined that $23.4 \%$ of the parents spent time with their family and were interested in activities and hobbies, $20.8 \%$ did nothing or tried not to think about the pandemic. When the parents' predictions about the pandemic were evaluated, it was seen that $33.8 \%$ of the parents stated that they would continue to take precautions even if the pandemic decreases. In addition, $33.2 \%$ of the parents stated that they believed there would be new pandemics, $19.5 \%$ believed that this pandemic would decrease over time, and $15.5 \%$ did not believe that the pandemic would decrease (Table 2).

When the effect of SFSP on parents' state anxiety scores was examined, a statistically significant difference was found between the pre-test $(56.10 \pm 10.14)$ and post-test $(43.51 \pm 9.22)$ STAI-S total scores of the intervention group to which the programme was applied $(\mathrm{p}=.000)$. However, there was no statistically significant difference between the pre-test and post-test STAI-S total scores of the control group $(\mathrm{p}=.616$, Table 3). When the changes in the parents' trait anxiety scores were examined, STAI-T post-test mean scores in the intervention group were found to be lower than the pre-test mean scores. However, this difference is not statistically significant $(\mathrm{p}=.149)$. In the control group, no statistically significant difference was found between the pre-test and post-test STAI-T mean scores $(\mathrm{p}=.817)$.

\section{SEM Test Results}

In the SEM, it is seen that SFSP decreases parents' anxiety $(\mathrm{p}=0.022$, Fig. 2$)$. The non-standard path coefficient of the STAI-S post-test was found to be 0.11 , and the obtained coefficient was statistically significant $(\mathrm{p}<0.05)$. The non-standard path coefficient of the STAI-T post-test was obtained as 0.03 and the obtained coefficient was statistically significant $(\mathrm{p}<0.05)$. When the fit values were examined, the following values were obtained: $\mathrm{CMIN}=7.639, \mathrm{DF}=2, \mathrm{CMIN} / \mathrm{DF}=3.819, \mathrm{RMSEA}=0.28, \mathrm{CFI}=0.865$ and $\mathrm{GFI}=0.913$. Since CFI, RMSEA, CMIN/DF were not within the required limits, the correction indices were examined.

\section{Discussion}

The burden and effects of COVID-19 are felt all around the world, and this is likely to cause fear and anxiety. ${ }^{36}$ This study was conducted with the aim of examining the effects of online SFSP applied to parents with high levels of anxiety during the COVID-19 pandemic. As a result of the study, it was determined that online SFSP was an effective method in reducing the state anxiety levels of parents with high anxiety levels.

In studies conducted with different sample groups in the COVID-19 pandemic, it has been reported that the pandemic significantly increases the anxiety level of children and parents. ${ }^{10,36}$ In this study, the anxiety levels of the parents of children aged 3-6 who had to spend most of their time at home by leaving the nursery, school and many social environments due to the pandemic were evaluated and it was determined that the pre-test anxiety levels of the parents were quite high in the intervention and control groups. In a study conducted in China $(\mathrm{n}=2446)$, it was stated that the participants had a high level of anxiety and approximately $80 \%$ of them scored above 40 in both STAI-S and STAI-T subscales. ${ }^{37}$ In the study conducted by Lee et al. (2020), it was determined that parents' anxiety levels were high and parental anxiety caused an increase in the anxiety level of children. ${ }^{10}$ In the study conducted by Saddik et al. (2020), it was determined that most of the participants experienced mild or severe anxiety due to the pandemic. In the same study, the participants stated that the measures taken by the government to prevent the pandemic increased their anxiety. ${ }^{24}$ In another study conducted with 1069 participants, it was stated that having a child is one of the reasons that increase anxiety during the COVID-19 pandemic. ${ }^{3}$ All these studies and our study findings show similarities in the sense that parental anxiety is high during the pandemic.

The COVID-19 pandemic places a wide range of stressors on young children, including its invisibility, the danger of infection, frustration and boredom, inadequate information, lack of face to face contact with family members, classmates, friends and teachers, and lack of personal space at home. Stress levels of children directly or indirectly cause stress and anxiety on their parents. ${ }^{38}$ In this study, more than half of the parents 
stated that their children's being away from the nursery and being constantly isolated at home made them aggressive and worsened their mental health. Sudden changes in the routines of children, who previously attended a nursery or nursing facility, events they cannot understand in a home-oriented life environment and many other factors can play a role in increasing the stress and anxiety levels of children and parents.

It has been reported that correct information about the virus has a negative and significant correlation with individuals' anxiety levels. ${ }^{39}$ In this study, almost all of the parents stated that they had access to information about COVID-19 via television $(44.2 \%)$ and social media $(37.8 \%)$. In the study conducted by Malesza and Kaczmarek (2021), more than $80 \%$ of the participants stated that they had access to COVID-19 information through television while $70 \%$ of the participants acquired information via the internet. However, only $20 \%$ of the participants had access to information through health authorities. When these findings are interpreted together with the high anxiety levels of the parents, it is thought that reaching the information from the right source may also have an effect on the anxiety level.

The global COVID-19 pandemic is a stressor that originated outside of the family system but given the novelty and uncertainty concerning this disease, it is likely to be perceived as a significant stressor for many parents and children. ${ }^{14}$ Individuals use different methods in order to cope with the anxiety experienced in the pandemic. When the coping behaviours of parents are examined; it is seen that they use different methods such as spending time with the family, trying not to think about the pandemic, cleaning, spending time by watching television and using telephone and praying. However, the high anxiety score averages of the parents indicate that these coping methods are not very effective in the pandemic process, which has very devastating effects.

It has been reported that the COVID-19 pandemic negatively affects the mental health of both parents and children and significantly reduces their psychological well-being. ${ }^{11,40}$ In our study, majority of the parents stated that they will continue to take measures even if the pandemic decreases and they believe that there will be new pandemic. These findings are important in terms of suggesting that parents experience anxiety about their future lives.

The effectiveness of SFSP in literature has been evaluated in many studies. ${ }^{17,29,41-43}$ Studies have shown that SFSP is effective in individuals with social anxiety disorder, ${ }^{16}$ reduces depression and anxiety, ${ }^{15,18}$ improves quality of life ${ }^{17}$, affects mindfulness and psychological well-being ${ }^{44}$ and is effective in solving mental problems by contributing to the development of all areas of mental health. ${ }^{43}$ In the study conducted by Novella, Ng and Samuolis (2020), SFSP was applied to university students with mild and moderate anxiety simultaneously with two different methods using face-to-face and online programmes. As a result of the study, it was determined that both methods significantly reduced students' anxiety levels and there was no significant difference between the effectiveness of the two methods. ${ }^{23}$ The use of video teleconferencing platforms is important in terms of providing a suitable alternative for continuity of care in times of social, economic and health-related problems, and provides more flexibility and access opportunities than face-to-face therapy. ${ }^{45}$ In our study, SFSP was applied through the online video-conference method due to the restrictions and precautions during the pandemic and it was found to be an effective method in reducing anxiety.

In order to reduce the anxiety level of individuals in the COVID-19 pandemic, different interventions were implemented and their effectiveness was evaluated. Internet-based integrated intervention focusing on relaxation, self-care, and raising sense of security applied to individuals diagnosed with COVID-19 with psychological problems was found to have a significant pleasing effect on mild and moderate depression and anxiety symptoms. ${ }^{46}$ In a study conducted, it was determined that informing via teleconference was effective in reducing the anxiety levels of mothers with chronic diseases during the pandemic period. ${ }^{36}$ In the study conducted by Chen (2020), it was aimed to administer 2-4 sessions of online Solution Focused Brief Therapy to adolescents who showed anxiety symptoms in the COVID-19 pandemic. It was hypothesized that participants who are randomly assigned to Solution Focused Brief Therapy would have betterclinical outcomes, lower depression levels, and increased coping strategies in dealing with distress during the COVID-19 pandemic. ${ }^{30}$ In our study, SFSP was applied to parents who had high levels of anxiety and it was found that the state anxiety levels of the parents were significantly reduced. In the model established in SEM analysis, 
it was determined that the SFSP intervention had a significant effect on anxiety (Fig. 2). After SFSP, the state and trait anxiety mean scores of the intervention group decreased compared to the pre-intervention mean scores. While this difference between state anxiety scores was statistically significant, the difference between trait anxiety scores was not statistically significant. With these findings, our first hypothesis was confirmed, while our second hypothesis was rejected. This result can be related to the fact that the state anxiety level is a result of the individual's direct psychological reactions related to negative events while the trait anxiety is related to the tendency to exhibit personal anxiety and it is relatively stable. This finding can be considered as an expected result, since the change in the state anxiety level must gain continuity in order for the trait anxiety level to change. However, it is noteworthy that the state anxiety score in the intervention group is still high (over 40) even in the post-test. It is an expected result that the anxiety does not decrease to the desired level due to the continuation of the pandemic.

\section{Limitations}

This study has several limitations. Firstly, the study results can only be generalized to the study population; however, the results cannot be generalized to all parents in Turkey. Inclusion of only parents with internet and computer infrastructure is an important limitation of the study. The pandemic continues worldwide. Parents' anxiety levels are likely to differ at the beginning or end of the pandemic. For this reason, the results should be interpreted against the background of the situation in Turkey at the time the study was conducted. Finally, SFSP was applied as 4 sessions in this study. The effect of SFSP on the trait anxiety levels of the participants can be investigated by increasing the number of sessions in future studies which will be applied to reduce anxiety.

\section{Conclusions}

As far as we know, this is the first study which investigates the effect of SFSP applied to parents with high level of anxiety in the COVID-19 pandemic on reducing anxiety. In the study, it has been found that SFSP applied to parents with high level of anxiety is an effective method in reducing the state anxiety levels of parents. Considering the advantages of applying SFSP in a short time and its rapid effect, it is recommended that SFSP should be applied to disadvantaged groups such as vulnerable children (with chronic diseases) and their parents in order to minimize the negative effects that may occur during and after the pandemic process. It may be recommended to provide in-service training on SFSP to people who provide services to the community (such as pediatric nurses, social workers, family health nurses). In addition, it is recommended to conduct follow-up studies to examine the long-term effects of SFSP.

Acknowledgements: The authors would like to thank all the parents who contributed by participating in the study

Conflict of Interest: No conflict of interest was declared by the authors.

Financial Disclosure: The authors declared that this study has received no financial support.

Data Availability Statement: The data that support the findings of this study are available from the corresponding author upon reasonable request.

\section{References}

\section{Hosted file}

Figure 1.pdf available at https://authorea.com/users/443986/articles/543852-the-effect-ofonline-solution-focused-support-program-on-parents-with-high-level-of-anxiety-in-thecovid-19-pandemic-a-randomized-controlled-study

\section{Hosted file}


Figure 2.pdf available at https://authorea.com/users/443986/articles/543852-the-effect-ofonline-solution-focused-support-program-on-parents-with-high-level-of-anxiety-in-thecovid-19-pandemic-a-randomized-controlled-study

\section{Hosted file}

Tables.pdf available at https://authorea.com/users/443986/articles/543852-the-effect-ofonline-solution-focused-support-program-on-parents-with-high-level-of-anxiety-in-thecovid-19-pandemic-a-randomized-controlled-study 